\title{
ALGUNOS PROBLEMAS DE LA TRADUCCIÓN DE LA TERMINOLOGÍA ONÍRICA GRIEGA AL CASTELLANO*
}

Carmen Barrigón y J. M. ${ }^{\mathrm{a}}$ Nieto

Universidad de Valladolid

El fin de una buena traducción es ser fiel transmisor de esencias y formas entre el autor y el lector, sin adiciones ni disminuciones, aunque a veces la lengua propia se nos presente como insuficiente para transmitir al contenido expresado por otra lengua.

Este problema adquiere especial relieve en obras de carácter técnico, donde la precisión en la expresión de los contenidos es fundamental. En estos casos no es fácil encontrar términos que permitan verter el contenido del original dentro de los propios moldes de la lengua receptora.

Y, en efecto, problemas de este tipo plantea también la traducción al castellano de los textos de la literatura griega antigua ${ }^{1}$. Así, desde el primer contacto

* Este artículo, con algunas modificaciones, se corresponde a la comunicación que con el título "La traducción de la terminología onírica: de Artemidoro de Daldis a Sigmund Freud", fue presentada en las Segundas Jornadas Nacionales de Historia de la Traducción, celebradas en León entre los días 29 y 31 de mayo de 1990.

1 Para todas estas cuestiones relativas a la problemática de la traducción, cfr. J. S. LASSO DE LA VEGA «La traducción de las lenguas clásicas al español como problema», Actas del III C.E.C.E., vol. I, Madrid 1968, pp 89-140, y V. Garcla YeBrA, En torno a la traducción (Teoría. Crítica. Historia), Madrid 1983. 
con la obra de Artemidoro de Daldis, La interpretación de los sueños ${ }^{2}$, nos han surgido serias dificultades de traducción precisamente en las palabras claves del libro, a saber, en la terminología específica de que se sirve el autor para denominar los diferentes fenómenos oníricos.

Por ello, este artículo tratará de perfilar las posibles traducciones de estos términos griegos al castellano a través de la comparación con otras lenguas de nuestro ámbito occidental, y con otras traducciones de obras oníricas al castellano, como es la obra de S. Freud, Die Traumdeutung. Por el momento nos vamos a central exclusivamente en los tres términos griegos básicos de materia

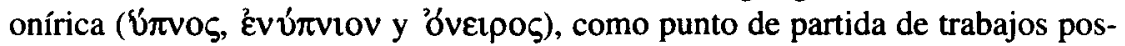
teriores sobre el tema.

Puesto que Artemidoro de Daldis es la figura clave a partir de la cual plantearemos los problemas específicos que presenta la traducción de la terminología onírica, comenzaremos de forma breve por situarle cronológicamente y esbozaremos algunos rasgos de su obra, remitiéndonos para un estudio amplio de estas cuestiones a cualquiera de las introducciones de las traducciones modernas de Artemidoro que veremos más adelante.

Las noticias que poseemos de este autor son escasas y se desprenden de su propia obra. Nos dice que nació en Éfeso, aunque prefiere proclamarse oriundo de Daldis, pequeña localidad de Lidia. Las referencias internas de su obra nos llevan a situarle cronológicamente en la segunda mitad del siglo II p. C. De su producción literaria únicamente se han conservado cinco libros de su Onirocriticon, aunque hay noticias de que compuso otras obras: él mismo dice que redactó escritos sobre teoría oniromántica y el Léxico de Suda le atribuye dos títulos: uno sobre adivinación mediante aves y otro sobre quiromancia.

Su obra consiste en una tarea de recopilación de las teorías anteriores ${ }^{3}$ con la incorporación de elementos nuevos a la investigación oniromántica, a saber, la experiencia y los casos prácticos ( $c f r$. el libro V). No olvidemos que el hombre griego se sintió atraído por la incógnita que representaba el sueño hasta el punto que se establecieron varias corrientes de especulación encabe-

2 En el Departamento de Filología Clásica de la Universidad de Valladolid estamos preparando una traducción al castellano de la obra mencionada de ARTEMIDORO DE DALDIS, que aparecerá publicada próximamente en la Editorial Akal en su colección de Clásicos Griegos.

3 Para poder calibrar la importancia de la investigación onírica en la Antigüedad pueden consultarse los excelentes trabajos de Th. HOPFNER «Traumdeutung", RE 12, cols. 2.233-2.245, $\mathrm{H}$. KENNER «Oneiros», RE 17, cols. 448-459, B. BUCHSENSCHUTZ, Traum und Traumdeutung im Alterthume, Berlín 1868 (reimpr. Wiesbaden 1967²), O. HEY, Der Traumglaube der Antike, Munchen 1908, D. BASSI «I sogni nei tragici greci», Aevum 17, 1943, pp. 237-241, y D. Del CoRno «Richerche sull'onirocritica greca» (Estrato dai Rendiconti, Classe di Lettere 96, 1962, pp. 334-366), Milano 1962. 
zadas por Platón, Aristóteles, y aquélla que se centra en el fenómeno del sueño como un hecho mántico, corriente encabezada fundamentalmente por el Estoicismo ${ }^{4}$.

De la doctrina onírica de Artemidoro lo fundamental para nuestro trabajo es su clasificación de los sueños. Siguiendo la idea tradicional divide los sueños entre los que tiene valor profético ('óveipo $\varsigma$ ) y los que carecen de un valor premonitorio (Évúrviov). A su vez, los 'óveıpol se dividen en directos, los que anuncian sucesos de inmediato, y simbólicos, los que tienen un cumplimiento retardado. Éste último es el que más le interesa a Artemidoro, dado que da pie a múltiples interpretaciones de tipo profético ${ }^{5}$ :

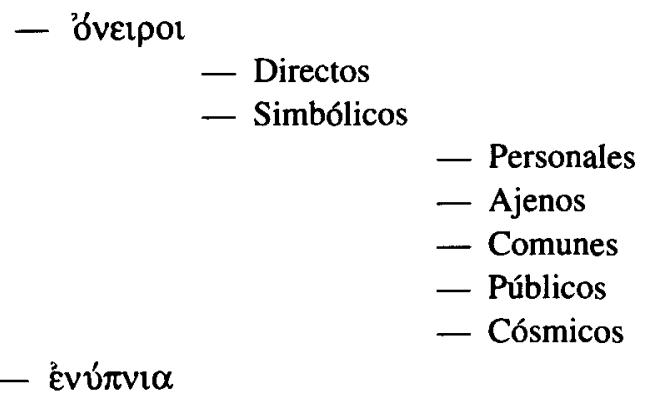

Para concluir esta breve introducción diremos que el mayor testimonio de la importancia de Artemidoro es Sigmund Freud, padre del psicoanálisis, quien atraído por el mundo griego, en su tan conocida obra, La interpretación de los sueños, menciona repetidamente a nuestro autor, destacando de Artemidoro el hecho de tener en cuenta la personalidad y circunstancias de la persona que tiene el sueño objeto de la investigación ${ }^{6}$.

${ }^{4}$ Cfr. D. DEL CoRno, Graecorum de re onirocritica scriptorum reliquiae, Milano-Varese 1969. E. SUÁREZ DE LA TORRE «El sueño y la fenomenología onírica en Aristóteles», Cuadernos de Filología Clásica, 5, 1972, pp. 279-311, y H. WIJSENBEEK-WuLER, Aristotle'concept of Soul, Sleep and Dream, Amsterdam 1978.

5 Interesante trabajo para las clasificaciones de los sueños es el de A. H. KESSELS «Ancient systems of dream-classification», Mnemosyne 22, 1969, pp. 389-424.

6 Para una comparación de la doctrina griega y la freudiana en materia de sueños es de gran utilidad la obra de A. BRAvo «La interpretación de los sueños: onirocrítica griega y análisis freudiano» en Tradición clásica y siglo XX, Madrid 1986, pp. 125-141. 
Tras esta pequeña panorámica de la personalidad y obra de Artemidoro pasamos ahora a la parte práctica de esta comunicación.

Parece evidente que la lengua castellana resulta insuficiente para recoger con vocablos precisos la variedad de los términos griegos en materia onírica.

Como es sabido la palabra castellana «sueño» encierra en sí misma dos realidades diferentes: el hecho de dormir y el hecho de soñar. El mismo término, por tanto, adquiere según el contexto un significado u otro. Resulta curioso, además, que el castellano presente esta carencia en el caso del sustantivo, y, en cambio, marque la distinción en el verbo: efectivamente, existe «dormir» y «soñar», pero sólo existe «sueño».

Por otra parte, el resto de las lenguas del ámbito occidental próximo distingue perfectamente en su vocabulario el sueño como fenómeno onírico del sueño como acto de dormir:

\begin{tabular}{lll}
\hline Alemán & Traum & Schlaf \\
Francés & Rêve & Sommeil \\
Inglés & Dream & Sleep \\
Italiano & Sogno & Sonno \\
\hline Castellano & \multicolumn{2}{c}{ Sueño } \\
\hline
\end{tabular}

De esta forma, es frecuente ver en cualquier traducción al castellano de una obra de contenido onírico la correspondiente nota del traductor señalando esta dificultad, y precisando el término o términos castellanos por los que ha optado para recoger cada vocablo original. Basta con abrir una de las primeras páginas de la traducción de La interpretación de los sueños de Sigmund Freud, donde el traductor López-Ballesteros ${ }^{7}$, por ejemplo, opta por el término «sueño» para el fenómeno onírico y «reposo» para el acto de dormir.

En consecuencia, el mismo problema con que se encuentra un traductor de Freud al castellano se lo plantea también el traductor de Artemidoro. Tal es el caso de la primera versión completa a nuestra lengua de esta obra, publicada el pasado año en la editorial Gredos, según veremos más adelante ${ }^{8}$.

${ }^{7}$ S. FREUd, La interpretación de los sueños, traducción de L. LOPEz-BALlESTEROS, Madrid 1981.

8 ARTEMIDORo, La interpretación de los sueños, introducción, traducción y notas de E. RuIZ GARCfA, Madrid 1989. 
Para ilustrar nuestra comunicación vamos a presentar el comienzo del libro I del Onirocriticon de Artemidoro ${ }^{9}$, y las traducciones que de él se han hecho en francés ${ }^{10}$, inglés ${ }^{11}$, italiano ${ }^{12}$ y castellano. El comienzo del libro 1 está dedicado a la clasificación de los sueños. Para Artemidoro los 'óveıpor son los sueños que tienen un valor profético; en cambio, los Ẻvítvi $\alpha$ carecen de un mensaje premonitorio. La obra de Artemidoro se centra exclusivamente en los 'óveıpol que se dividen y subdividen a su vez en sucesivos tipos.

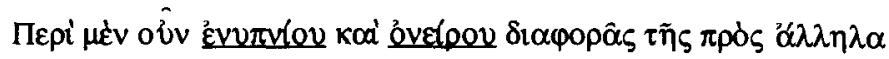

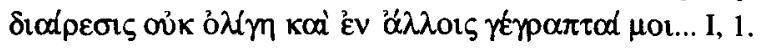

Veamos las correspondientes traducciones:

«Eh bien donc, touchant la différence mutuelle entre rêve et vision de songe, la distinction n'est pas médiocre et j'en ai traité ailleurs...» ${ }^{13}$.

«The distinction, the, between enhypnion and oneiros is great, as I have explained in other works.... ${ }^{14}$.

«Riguardo alla differenza che intercorre reciprocamente tra la visione onirica e il sogno, ho giá esposto una distinzione particolareggiata in altre opere...» ${ }^{15}$.

«En lo que concierne a la diferencia existente entre ensueño y visión onírica, ya he expuesto en otras obras que una distancia no exigua los separa.... ${ }^{16}$.

${ }^{9}$ Para el texto griego seguimos la edición de R. A. PACK, Artemidori Daldiani Onirocriticon Libri V, Leipzig 1963.

10 Cfr. A. J. Festugière, Artémidore, La clef des songes, París 1975.

1 Cfr. R. J. WhITE, Artemidorus. The Interpretation of Dreams, New Jersey 1975.

12 Cfr. D. Del CoRno, Artemidoro, ll libro dei sogni, Milano 1975.

13 Cfr. Festugière, op. cit., p.19.

14 Cfr. WHITE, op. cit., p. 26.

15 Cfr. Del CORNO, op. cit., p. 5.

16 Cfr. Ruiz García, op. cit., p. 72. 
Veamos de forma gráfica estas diferentes traducciones:

\begin{tabular}{|c|c|c|}
\hline & 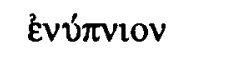 & 'óveıрos \\
\hline $\begin{array}{l}\text { Francés } \\
\text { Inglés } \\
\text { Italiano } \\
\text { Castellano }\end{array}$ & $\begin{array}{l}\text { rêve } \\
\text { enhypnion } \\
\text { visione onirica } \\
\text { ensueño }\end{array}$ & $\begin{array}{l}\text { vision de songe } \\
\text { oneiros } \\
\text { sogno } \\
\text { visión onírica }\end{array}$ \\
\hline
\end{tabular}

Como se desprende de este cuadro el problema de la traducción del griego se complica en Artemidoro al introducir en su clasificación el término

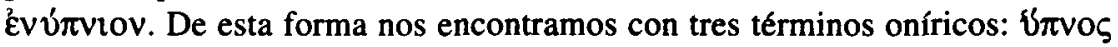

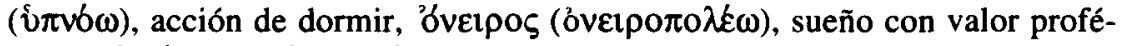

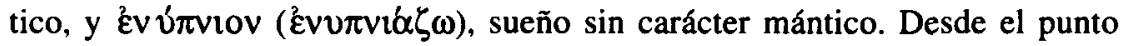
de vista de la lengua griega, en principio no existe diferencia semántica entre "óveıpos y žv úrviov, ambos términos hacen referencia a los sucesos que se producen durante el estado de reposo, y se emplean sinónimos ${ }^{17}$. Aristóteles en sus tratados sobre los sueños no distingue entre los dos vocablos ${ }^{18}$. Por tanto parece ser un aprovechamiento, desde el punto de vista semántico, de esta doble terminología por parte de Artemidoro para expresar su propia teoría onírica.

De esta forma la triple terminología onírica la expone Artemidoro en el siguiente párrafo de este primer libro:

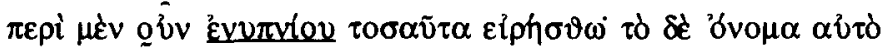

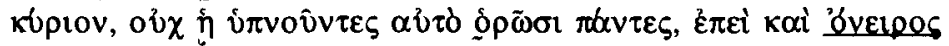

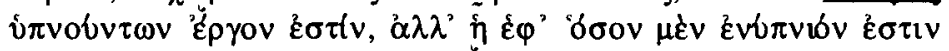

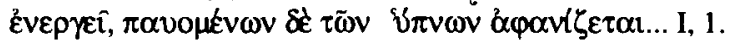

He aquí sus correspondientes traducciones:

${ }_{17}$ Así ocurre, por ejemplo, en Homero y Heródoto, vid. Il. 2.55 y Od. 14. 495; para Heródoto, vid. J. E. Powell, A Lexicon to Herodotus, Hildesheim, 1966.

18 En concreto para Aristóteles vid. Suárez, art. cit. 
«Touchant donc le rêve, en voilà assez. Quant au nom même de rêve pendant le sommeil, il est propre, non pas en tant que c'est toujours quand on est en sommeil qu'on rêve ainsi- car la vision de songe aussi est le fait de gens en sommeil- mais en tant que le rêve influe seulement dans la mesure où il se passe durant le sommeil, alors que, le sommeil achevé, il disparait.... ${ }^{19}$.

«So much, then, for the enhypnion. The name itself is significant, no insofar as all those who see it are asleep (since even oneiros is the product of sleepers), but insofar as the operation of the enhypnion is limited to the duration of one's sleep; the minute the sleeping ends, it disappears...» ${ }^{20}$.

«Tanto sia detto a proposito della visione onirica; e il suo nome risulta appropiato, non perché tutti la vedono durante il sogno, in quanto anche il sogno è un'attività di chi dorme: ma perché ha effetto fin tanto che dura il sonno, e scompare quando il sonno vien meno.... ${ }^{21}$.

«Baste con lo dicho sobre el ensueño. Su mismo nombre resulta apropiado no porque todos lo ven mientras que duermen- ya que también la visión onírica es una actividad propia de los que realizan esta acción- sino porque tiene vigencia mientras la persona permanece en estado de reposo.... ${ }^{22}$.

En consecuencia, la traducción del texto de Artemidoro evidencia la siguiente equivalencia en la terminología onírica:

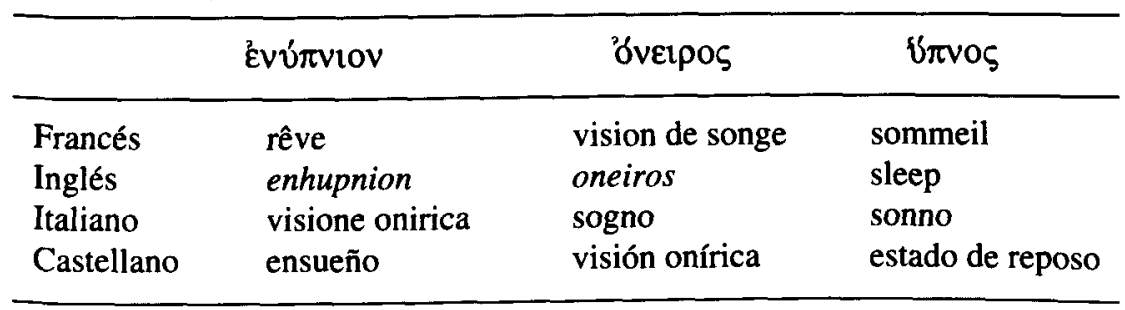

${ }^{19}$ Cfr. Festugtère, op. cit., pp. 19-20.

${ }^{20}$ Cfr. WhITE, op. cit., pp. $27-28$.

21 Cfr. Del CoRno, op. cit., p. 6.

22 Cfr. Ruiz Garcfa, op. cit., p. 74. 
Como vemos, cada traductor ha optado, de acuerdo con la capacidad léxica de su propia lengua, por diferentes términos para reproducir de la forma más fiel y precisa los conceptos griegos. La dificultad de esta empresa queda manifiesta en la versión inglesa de White, que no traduce los términos sino que prefiere dar su transcripción, al menos de los conceptos claves de la doctrina onírica.

Efectivamente, la traducción se complica por la inclusión de Artemidoro del vocablo évúrviov. Desde el punto de vista griego la etimología es clara,

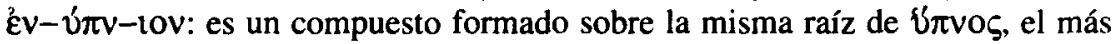
importante, con la preposición $\varepsilon^{2} v$ como primer elemento ${ }^{23}$. A pesar de su significado etimológico «lo que aparece en los sueños», sin embargo se emplea también con un sentido más amplio y similar a 'óvetpo $\varsigma^{24}$. No obstante, es una oposición léxica equivalente a la del latín, somnus frente a insomnium, o la del castellano «sueño» frente a «ensueño».

El francés y el italiano respetan de forma clara y precisa en sus traducciones esta clasificación tripartita sin necesidad de recurrir a términos que necesiten ser explicados en una nota del traductor. Precisamente es esto último lo que ocurre en la traducción de Artemidoro al castellano, que tiene que precisar ampliamente en una nota la correspondencia castellana de cada término griego.

Una vez vista la dificultad de adaptación y traducción de la terminología onírica a nuestra lengua, para finalizar simplemente nos queda ya proponer nuestra versión particular de estos conceptos de la obra de Artemidoro. Hemos optado por traducir $U \pi v 0 \varsigma$ por «estado de reposo», 'óvelpo guiendo así una tradición mantenida en las traducciones de Freud al castellano. Y finalmente हैvúitviov por «ensueño», a pesar de que el término castellano no es muy preciso en este contexto. Por tanto, en este último caso debemos precisar, en su correspondiente nota, que se trata de recoger, o mejor dicho, calcar etimológicamente el concepto griego de «visión del sueño» 0 «lo que ocurre en sueños».

No obstante, en todo caso nos veremos obligados también a precisar en sus correspondientes notas a pie de página nuestra particular versión de estos términos, y todo ello debido a la incapacidad de nuestra lengua para recoger toda la riqueza léxica de la lengua griega en el campo de la onirología.

${ }^{23}$ Cft. P. Chantraine, Dictionaire Étimologique de la langue grecque, París 1968, s. u. Uлvos.

24 Cfr. supra nota 15. 